\title{
RECONSTRUCTION OF A NEGLECTED LOCKED POSTERIOR FRACTURE - DISLOCATION OF HUMERAL HEAD: A CASE REPORT
}

Pavan H. M11, Muralidhar N.

HOW TO CITE THIS ARTICLE:

Pavan H. M. Muralidhar N. "Reconstruction of a neglected locked posterior fracture - dislocation of humeral head: a case report". Journal of Evolution of Medical and Dental Sciences 2013; Vol2, Issue 29, July 22; Page: 5388-5394.

ABSTRACT: BACKGROUND: Posterior shoulder dislocations and fracture-dislocations are uncommon injuries that most often occur during seizures or as a result of high-energy trauma. Despite advances in imaging, they are frequently diagnosed late. Detection is facilitated by heightened clinical suspicion of the injury in high-risk individuals together with appropriate radiographic investigation.

A wide variety of operative techniques, ranging from simple closed reduction to soft-tissue and bone stabilization procedures to prosthetic arthroplasty, are available to treat these injuries. Selection of the most appropriate treatment option is complex and multifactorial.

Good functional outcomes are associated with early detection and treatment of isolated posterior dislocations that are associated with a small osseous defect and are stable following closed reduction. Poor prognostic factors include late diagnosis, a large anterior defect in the humeral head, four part fracture or arthrosis of the humeral head.

CASE DESCRIPTION: A 24 year old male worker presented with persisting pain and stiffness in his right shoulder following high energy trauma 8 months back. Physical examination and radiological evidence showed posterior dislocation with humeral head fracture. Patient was treated by open reduction and reconstruction of the proximal humerus. Post operative follow up for 18 months showed good range of movements. Early diagnosis and treatment of this rare type can prevent deformities.

INTRODUCTION: Posterior locked shoulder dislocation is an uncommon injury $(2-4 \%$ of all shoulder dislocations) which may be misdiagnosed and overlooked in up to $60 \%$ of cases. ${ }^{1}$ The spectrum of associated injuries varies from the isolated impaction fracture of the anteromedial aspect of the humeral head ("reverse Hill-Sachs lesion") to more complex fracture types of the proximal humerus (less than 1\%) and shoulder girdle 1,2. The unrecognized dislocation-fracture pattern can jeopardize the joint mobility and the vascularity of the humeral head predisposing to chronic instability, osteonecrosis and osteoarthritis .1 The management of posterior fracture dislocation after delayed diagnosis is complicated. ${ }^{3}$

CASE PRESENTATION: A 24-year-old right-hand dominant male worker, presented at the Vydehi Hospital complaining of persisting pain and stiffness in his right shoulder. The symptoms began 8 months earlier following a high energy trauma. The patient reported that he had taken native treatment with an osteopath where serial massages were done to relieve the pain, as there was no improvement in pain and shoulder mobility; he finally was referred to our hospital for a second opinion and further evaluation. 


\section{CASE REPORT}

On physical examination, his shoulder looked flattened anteriorly and both acromion and coracoid processes appeared to be prominent at the anterior part of the shoulder. There was an internal rotation deformity of $20^{\circ}$ and any effort to passively or actively move the glenohumeral joint was extremely painful. Forward elevation of $40^{\circ}$, no external rotation and inability to completely supinate the forearm were also identified. The patient did not have any neuromuscular deficit and he had no other medical comorbidities.

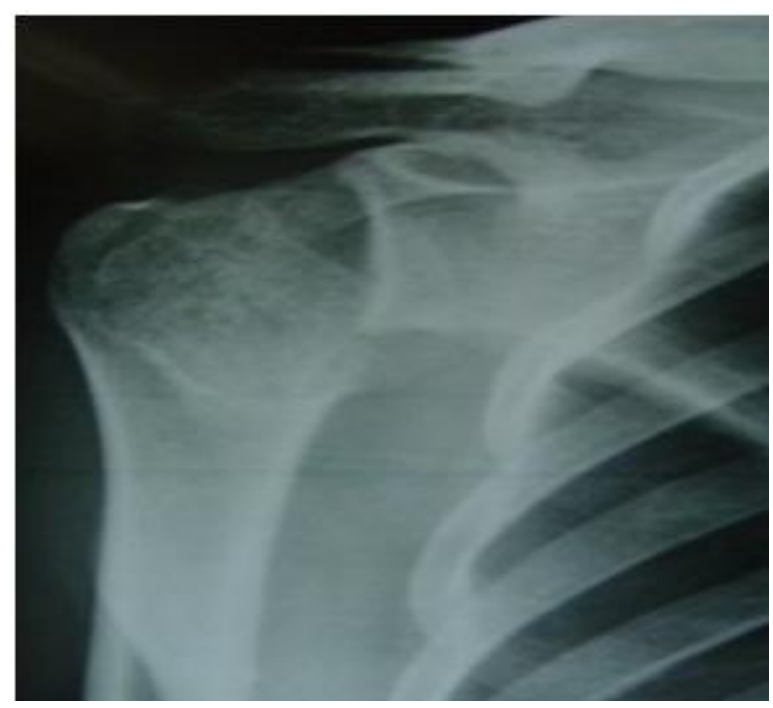

fig 1-A : Anteroposterior radiograph of the right shoulder of 24 year old man,8 months past injury illustrated the marked internal rotation of the proximal humerus and the typical "light bulb sign".

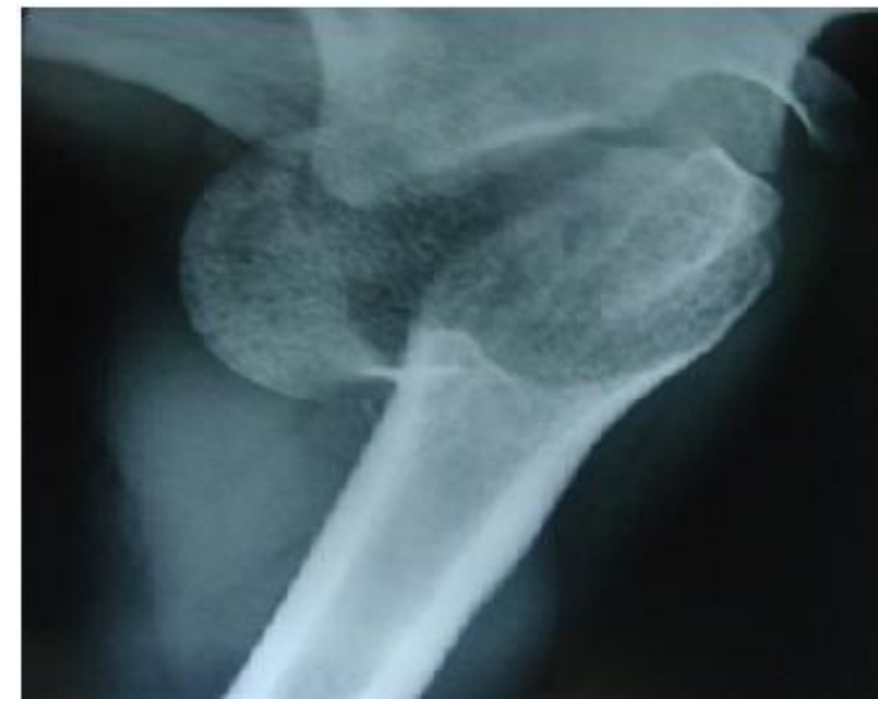

Fig 1-B: Axillary lateral radiographs showing posterior dislocation with humeral head fracture.

According to these findings, open reduction and reconstruction of the proximal humerus was considered necessary. Under general anaesthesia, the patient was placed in a beach chair position 
and the glenohumeral joint was assessed via a deltopectoral approach. The axillary nerve was protected, careful osteoclasis was done As the capsule was torn and detached along with the lesser tuberosity, mobilization of the bone fragment in a "trap-door" manner allowed easy access and visualisation of the glenohumeral joint. The humeral head was found to be dislocated posteriorly, After meticulous removal of the scar tissue, the glenoid articular cartilage looked to be in good condition along with the humeral head .Anatomic neck fracture was stabilized with cannulated screws and found to be stable through the range of movements. The reverse Hill-Sachs lesion was addressed with transfer of the fractured lesser tuberosity and its attached subscapularis muscle to the anteromedial defect according to McLaughlin's technique modified by Hawkins . ${ }^{3}$ Aiming to restore the sphericity of the humeral head and enhance the healing process, the bone bed of the defect was augmented with demineralized bone matrix allograft. Repair of the rotator interval was the last step performed and routine closure of the wound over a drain was achieved.
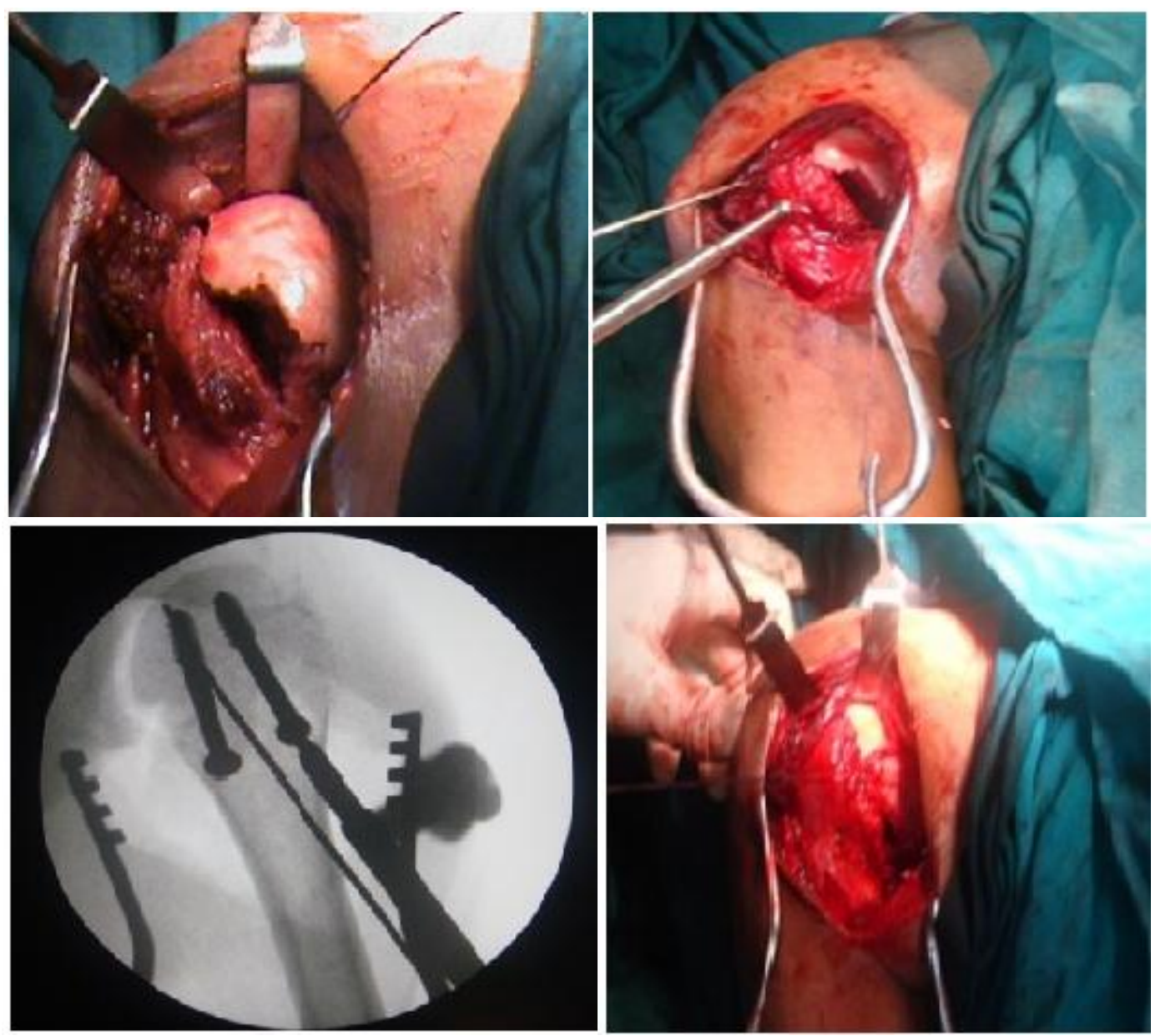

Fig 2 : Intraoperative photographs of right shoulder showing humeral head after reduction with reverse Hill-Sachs lesion( about 25\%), fracture fixation with partially threaded screws, transfer of subscapularis to anteromedial defect (McLaughlin's technique modified by Hawkins). 


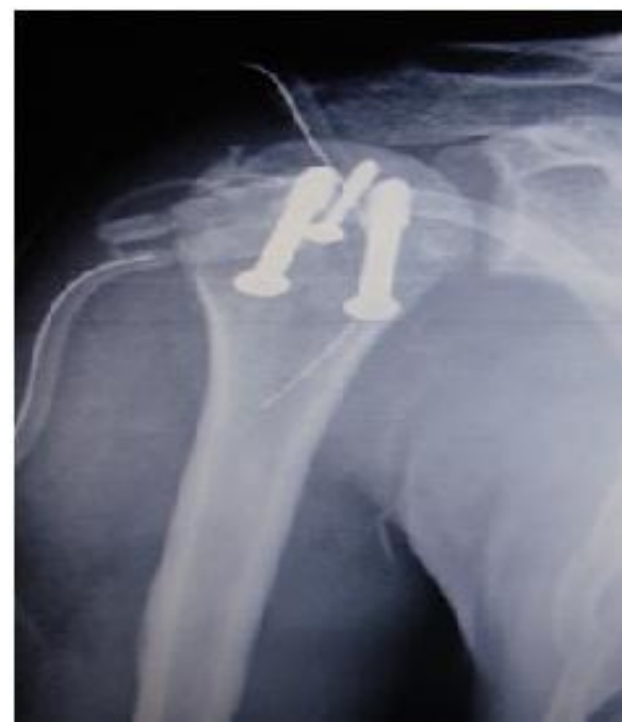

Fig 3: Postoperative radiograph showing good reduction of fracture.

Postoperatively, the extremity was placed in a sling with the shoulder in neutral rotation and slight abduction. At 4 weeks, passive shoulder and pendulum exercises were initiated and the patient was advised to use the sling for another 4 weeks. At 8 weeks, a more aggressive physical therapy with active assisted range-of-motion and strengthening exercises was instituted as plane Xrays showed maintenance of joint congruency and early signs of bone healing. He reported that his shoulder was totally painless without any limitations during daily activities. He could actively elevate and abduct his arm $150^{\circ}$ and $120^{\circ}$, respectively. In internal rotation, he reached the L2 vertebra and external rotation was $40^{\circ}$. Plane radiography confirmed a good clinical result and absence of devascularisation or instability of the humeral head.

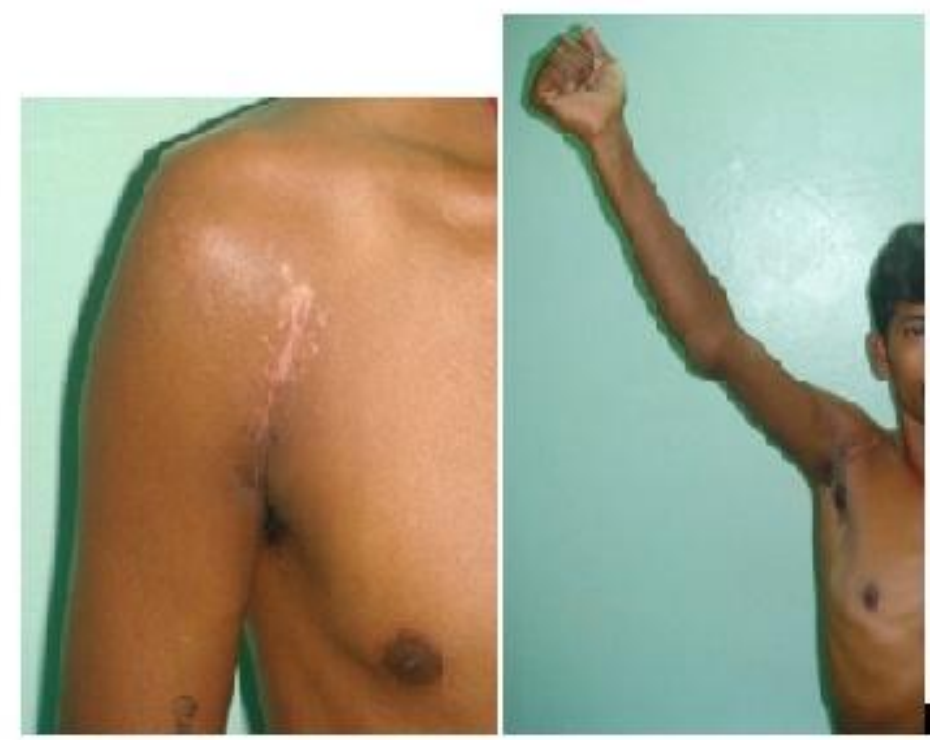

Fig 4: Follow up 18 months postoperatively showing good range of movements.

DISCUSSION: The rarity of incidence of posterior-fracture dislocation, the potential for delay in diagnosis and the lack of evidence-based management strategies make this specific injury type 
challenging to treat. Recently, Robinson et al. ${ }^{2}$ divided posterior-fracture dislocations into three subtypes according to the extent of fracture lines and the involvement of tuberosities. In Type I, a Neer Two-Part anatomic fracture is present without associated tuberosity fractures. In Type II, there is an additional fracture of the lesser tuberosity and in rare Type III both tuberosities are involved.

Apart from the severity of injury and fracture deformity, the final prognosis is further affected by the extent of the underlying glenoid or reverse Hill-Sachs lesion ${ }^{4}$ 5; the focus is concentrated on treatment of the anteromedial defect of the humeral head. Transfer of the subscapularis or lesser tuberosity, rotational osteotomy of the humerus and allograft or autograft reconstruction have been advocated for the treatment of medium (25-40\% of articular surface) or large (more than 40\%) defects in cases where the articular cartilage has been impressed but not destroyed ${ }^{4}$. Hemiarthroplasty has been suggested in patients with an impression fracture involving more than $50 \%$ of the articular surface or when the humeral head is very soft and not viable .However, in young patients, all efforts should be made to retain the humeral head and restore its shape, roundness and normal anatomy. Similar to our case, good results have been reported after reconstruction of defects equal to or greater than $40 \%$ of the articular surface using allograft or lesser tuberosity transfer 7,8 .

The transfer of lesser tuberosity instead of subscapularis alone was first introduced by Hawkins et al. 3 Finkelstein et al ${ }^{9}$ reported that full flexion, abduction, and external rotation were achieved at 3 months in seven acutely treated shoulders with a $20 \%$ to $45 \%$ humeral head defect. The authors stated that the technique allowed earlier joint mobilization because of the increased confidence in the immediate stability of the repaired shoulder. Checchia et al. ${ }^{10}$ noted similar results but emphasised the importance of the time interval between injury and diagnosis. Specifically, posterior fracture-dislocations which were treated within 2 years of the injury had good shoulder function in comparison with neglected and misdiagnosed cases.

Although avascular necrosis of the humeral head is unpredictable, accurate reduction and stable internal fixation - even if performed late - enhance the probability of successful revascularization of the humeral head and avoid the development of avascular necrosis ${ }^{11}$. Head reperfusion seems to occur by the intact posteromedial vessels or alternatively by "creeping substitution" in cases with severe disruption of the arterial flow and soft tissue attachments ${ }^{6}$. In the presented case, the impaction of demineralized bone matrix might contribute to the viability of humeral head due to its osteo conductive and osteo inductive properties. Even though it does not offer structural support, it is well suited for filling bone defects and cavities and it can be revascularized quickly. We believe that transposition of lesser tuberosity combined with allograft impaction can effectively address large humeral defects and decrease the potential of subchondral collapse or avascular necrosis.

CONCLUSION: Posterior shoulder fracture-dislocation continues to be a "diagnostic trap" for the unaware physician despite the advances in imaging techniques and the continuous flow of information about the risk of missed diagnosis. The presented case underlines the variability of injury pattern, the potential of missed diagnosis and the need for preserving the humeral head in young patients regardless of the amount of articular surface defect and disruption of soft tissue attachments. 


\section{REFERENCES:}

1. Bock P, Kluger R, Hintermann B: Anatomical reconstruction for Reverse Hill-Sachs lesions after posterior locked shoulder dislocation fracture: a case series of six patients. Arch Orthop Trauma Surg 2007, 127(7):543-548.

2. Robinson CM, Akhtar A, Mitchell M, Beavis C: Complex posterior fracture-dislocation of the shoulder. Epidemiology, injury patterns, and results of operative treatment. J Bone Joint Surg Am 2007, 89(7):1454-1466.

3. Hawkins RJ, Neer CS 2nd, Pianta RM, Mendoza FX: Locked posterior dislocation of the shoulder.

J Bone Joint Surg Am 1987, 69(1):9-18.

4. Edelson G, Kelly I, Vigder F, Reis ND: A three-dimensional classification for fractures of the proximal humerus.

J Bone Joint Surg Br 2004, 86(3):413-425.

5. Rowe CR, Zarins B: Chronic unreduced dislocations of the shoulder. J Bone Joint Surg Am 1982, 64(4):494-505

6. Robinson CM, Aderinto J: Posterior shoulder dislocations and fracture-dislocations. J Bone Joint Surg Am 2005, 87(3):639-650.

7. Delcogliano A, Caporaso A, Chiossi S, Menghi A, Cillo M, Delcogliano M: Surgical management of chronic, unreduced posterior dislocation of the shoulder. Knee Surg Sports Traumatol Arthrosc 2005, 13(2):151-155.

8. Gerber C, Lambert SM: Allograft reconstruction of segmental defects of the humeral head for the treatment of chronic locked posterior dislocation of the shoulder.

J Bone Joint Surg Am 1996, 78(3):376-382.

9. Finkelstein JA, Waddell JP, O'Driscoll SW, Vincent G: Acute posterior fracture dislocations of the shoulder treated with the Neer modification of the McLaughlin procedure. J Orthop Trauma 1995, 9(3):190-193.

10. Checchia SL, Santos PD, Miyazaki AN: Surgical treatment of acute and chronic posterior fracture-dislocation of the shoulder. J Shoulder Elbow Surg 1998, 7(1):53-65.

11. Kaar TK, Wirth MA, Rockwood CA Jr.: Missed posterior fracture-dislocation of the humeral head. A case report with a fifteen-year follow-up after delayed open reduction and internal fixation.

J Bone Joint Surg Am 1999, 81(5):708-710. 


\section{AUTHORS:}

1. Pavan H.M.

2. Muralidhar N.

\section{PARTICULARS OF CONTRIBUTORS:}

1. Assistant Professor, Department of Orthopaedics, Vydehi Medical College.

2. Professor \& Head, Department of Orthopaedics, Vydehi Medical College.

\section{NAME ADRRESS EMAIL ID OF THE} CORRESPONDING AUTHOR:

Dr. Pavan H.M, Assistant Professor, Department of Orthopaedics, Vydehi Medical College, Bangalore. Email: drpavanhm81@rediffmail.com

Date of Submission: 14/07/2013.

Date of Peer Review: 15/07/2013.

Date of Acceptance: 16/07/2013.

Date of Publishing: 18/07/2013 